\title{
Enthalpy versus entropy: what drives hard-particle ordering in condensed phases?
}

\section{July 2016}

Authors: Mitchell Anthamatten ${ }^{1,2, a)}$, Jane J. Ou ${ }^{1}$, Jeffrey A. Weinfeld ${ }^{1}$, Shaw H. Chen ${ }^{1,2, a)}$

\begin{abstract}
Affiliations:
${ }^{1}$ Department of Chemical Engineering, University of Rochester, Rochester, New York, 146270166, USA.

${ }^{2}$ Laboratory of Laser Energetics, University of Rochester, Rochester, New York, 14623-1212, USA.

${ }^{a}$ Authors to whom correspondence should be addressed. Electronic mail: anthamatten@che.rochester.edu,shch@1le.rochester.edu
\end{abstract}




\begin{abstract}
In support of mesoscopic-scale materials processing, spontaneous hard-particle ordering has been actively pursued for over a half-century. The generally accepted view that entropy alone can drive hard particle ordering is evaluated. A thermodynamic analysis of hard particle ordering was conducted and shown to agree with existing computations and experiments. Conclusions are that (i) hard particle ordering transitions between states in equilibrium are forbidden at constant volume but are allowed at constant pressure; (ii) spontaneous ordering transitions at constant pressure are driven by enthalpy, and (iii) ordering under constant volume necessarily involves a non-equilibrium initial state which has yet to be rigorously defined.
\end{abstract}


The interdisciplinary topic of molecular self-assembly has provided fundamental insight into important phenomena such as micelle formation, protein folding, and molecular crystallization. Currently, there is intensive effort to harness nanoscale particles' innate ability to self-assemble, thereby enabling the design and processing of advanced nanomaterials with potential applications in biomedicine, catalysis, optoelectronics, and renewable energy. Selfassembly is a complex and delicate process, and the outcome of ordering depends on the participating particles' shape, site-specific binding forces, the type of medium, and the presence of interfaces and external fields. Nonetheless, the thermodynamic properties of many atomic, molecular, and nanoscale systems can be reasonably well represented by hard-particle models despite their size disparity. Here, we consider ordering transitions that encompass isotropic liquids, thermotropic liquid crystals, and crystalline solids without suspending liquids upon cooling or compression, while excluding glass transitions where vitrification does not contribute to ordering.

The possibility of entropy-driven hard particle ordering dates at least back to the prediction of lyotropic mesomorphism in colloidal suspensions of hard rods at high enough concentration. ${ }^{1}$ A signature of hard-sphere ordering first emerged through molecular dynamics and Monte Carlo calculations of the equation of state where crystallization was observed. ${ }^{2,3}$ These early findings have motivated over half a century of intensive research on the nucleation kinetics and equilibrium thermodynamics governing phase transitions of hard particles. It has been suggested that crystal structure and microstructure are largely determined by phase equilibrium and solidification pathway, respectively. ${ }^{4}$ The predominant view is that ordering to equilibrium final states is driven by an entropy increase at constant system volume. ${ }^{5-13}$ To properly evaluate the thermodynamic origin of hard-particle ordering, one must consider phase 
transition between two states in equilibrium as the foundation for devising nanoscale materials processing strategies.

As the first step, we will examine the equilibrium ordering transition by addressing two questions: (i) Are hard-particle ordering transitions allowable at constant volume? and (ii) Can entropy alone drive hard-particle ordering without incurring enthalpy? We will then comment on the more challenging problem of hard particle ordering from initial, less ordered states out of equilibrium with the final, better ordered states.

To answer the two questions raised above, consider the Helmholtz and Gibbs energy defined as $A=U-T S$ and $G=H-T S=U+P V-T S$, in which $P$ and $T$ are pressure and temperature, and $S, H, U$ and $V$ are entropy, enthalpy, internal energy, and volume of the system. A closed system permits energy exchange with its surroundings while consisting of a fixed number of particles, $N$, which are present as an arbitrary number of components and phases at uniform $T$. The criteria for equilibrium and process spontaneity for an ordering transition with a differential perturbation from equilibrium are $d A \leq 0$ at constant $(V, T)$ or, equivalently, $d G \leq 0$ at constant $(P, T) .{ }^{14}$ These criteria take into account both the first and the second laws of thermodynamics. Hard particles are characterized by the absence of interaction energy when they are separated but experience infinite repulsion upon contact in all physical states. As a result, temperature-dependent translational motion is solely responsible for kinetic energy with mass concentrated at the hard-particle centers. With $\Delta$ characterizing the finite property change, $\Delta U=0$ accompanies an ordering transition at constant $T$ regardless of $P$ and $V$. Therefore, equilibrium and process spontaneity can be evaluated using $\Delta A=-T(\Delta S) \leq 0$ at constant $(V, T)$ or $\Delta G=P(\Delta V)-T(\Delta S) \leq 0$ at constant $(P, T)$. 
Let us probe the thermodynamic origin of hard-particle ordering within the Gibbs energy framework using available computational and experimental results for ordering transitions under equilibrium at constant $(P, T)$, where $\Delta G=P \Delta V-T \Delta S=0$. Monte Carlo simulations of hard spheres in the single-phase regions under constant $(P, T)$ by Hoover and Ree ${ }^{15}$ resulted in crystallization at volume fractions $\phi_{\mathrm{M}}=0.494$ and $\phi_{\mathrm{X}}=0.545$ in transition from the isotropic melt to crystal phase. Their results have remained widely accepted with the coexistence pressure, $P_{\text {eq }}$, adjusted merely by $-1 \%$ in the thermodynamic limit, as suggested by subsequent Monte Carlo calculations nearly four decades later. ${ }^{16}$ Isothermal-isobaric Monte Carlo simulations were also performed for hard rods by McGrother et al. ${ }^{17}$ in the single-phase regions, revealing an isotropicto-nematic transition followed by a nematic-to-smectic A transition upon stage-wise compressions. Their computational results have been employed recently to help validate physics engine, a software framework for large-scale multibody simulations. ${ }^{18}$ The consistent observation that $\Delta V<0$ in isobaric simulations of hard particles requires that $\Delta H<0$ and $\Delta S<0$ as well. As one of the few experimental studies, Rutgers et al. ${ }^{19}$ conducted x-ray transmission densitometry on electrostatically screened, polystyrene spheres in 3 and $6 \mathrm{mM} \mathrm{HCl}$ aqueous solutions with the conclusion of a volume reduction at given $(P, T)$ accompanying the melt-tocrystal transition. Moreover, the melt and crystal volume fractions under equilibrium are identical to those by Hoover and Ree's Monte Carlo simulation. ${ }^{15}$ In addition, the experimental data reported by Rutgers et al. were employed to help uphold 0.64 as the universal maximum random close packing density with and without suspending liquids. ${ }^{20}$ The quantitative agreement between the computed and measured equations of state for hard spheres is by and large acceptable within experimental uncertainties established by Royall et al. ${ }^{21}$ : reproducibility 
to within $10^{-4}$ with a systematic error estimated at \pm 3 to $6 \%$ associated with the measurement of core volume fraction.

Having established that $\Delta S<0$ as a result of $\Delta V<0$ for ordering transitions of hard spheres and hard rods at constant $(P, T)$, let us proceed to quantitatively evaluate $\Delta S$ for a comparison between calculations and available experimental data. According to Lekkerkerker and Tuinier, ${ }^{22} P_{\text {eq }}=(6.12) k_{\mathrm{B}} T / v_{0}$ with $k_{\mathrm{B}}$ and $v_{0}$ denoting the Boltzmann constant and the hardsphere volume, respectively. Hoover and Ree ${ }^{15}$ offered an equivalent expression $P_{\text {eq }}=$ (8.27) $N k_{\mathrm{B}} T / V_{0}$ in the thermodynamic limit, where $V_{0}$ denotes the system volume at close packing. The two expressions are related through $v_{0}=\phi_{\mathrm{FCC}} V_{0} / N$ with $\phi_{\mathrm{FCC}}=0.741$ as the maximum volume fraction in a face-center-cube lattice. The volume and entropy loss associated with crystallization can be readily calculated using the hard-sphere equation of state. Across the melt-to-crystal transition, $\Delta V=N v_{0}\left(\phi_{\mathrm{X}}{ }^{-1}-\phi_{\mathrm{M}}{ }^{-1}\right)$ at a constant $P_{\text {eq. }}$. Upon crystallization from $\phi_{\mathrm{M}}=$ 0.494 to $\phi_{\mathrm{X}}=0.545$ under melt-crystal equilibrium, the system entropy changes by $\Delta S=\Delta H / T=$ $P(\Delta V) / T=-1.16 N k_{\mathrm{B}}$, in excellent agreement with $-1.17 N k_{\mathrm{B}}$ from molecular dynamics calculations. $^{23}$ The calculated entropy loss is also quite close to the experimental values from -1.61 to $-1.70 N k_{\mathrm{B}}$ for rare gases $\mathrm{Ne}$ through $\mathrm{Xe} .^{24}$ The agreement between calculations and experiments inspires our confidence in condensed spherical atoms being faithfully modeled as hard spheres. All existing computational and experimental data embrace $\Delta V<0$ and $\Delta S<0$ and hence $\Delta H=P \Delta V<0$, consistent with the outcome of Gibbs energy analysis as shown in Supporting Information.

From a broader perspective, the conclusion derived from single-component systems is applicable to binary mixtures of hard spheres, such as phase separation with simultaneous crystallization of the larger component. ${ }^{25}$ This process has been interpreted as entropy-driven. 
In fact, the underlying hard-particle crystallization takes place across the melt-crystal equilibrium at given $(P, T)$. Thus, the thermodynamic quantities $-V, S$ and $H$ - all experience a step reduction across the melt-to-crystal transition. Isothermal-isobaric Monte Carlo simulations have also been conducted to show that hard rods doped with hard spheres at 5 volume $\%$ undergo isotropic-tonematic and nematic-to-smectic phase transitions, each with an increased extent of demixing accompanied by a decreased total $V$ at constant $P$ without a suspending liquid. ${ }^{26}$ These two additional examples are readily understood in terms of $\Delta S=\Delta H / T=P(\Delta V) / T<0$ on the Gibbs energy platform.

To formally appraise the feasibility of hard-sphere crystallization across the two-phase region under equilibrium at constant $P$ versus constant $V$, the computed equation of state $^{15,23}$ is adapted in Figure 1a as $P / T=\mathrm{f}(V)^{10}$ as expected of hard-particle systems on physical grounds. Figure 1a indicates that the ratio $P / T$ has a distinct value at each $V$ in the single-phase (i.e. melt and crystal) region, while the ratio remains constant throughout the melt-crystal coexistence region, $V_{\mathrm{X}} * \leq V \leq V_{\mathrm{M}} *$, where $V_{\mathrm{X}} *$ and $V_{\mathrm{M}} *$ represent the system volume of crystal and melt on the verge of melting and crystallization, respectively. As demonstrated in Figure 1b, crystallization of a melt is completely forbidden at constant $V$, as a typical $V=V_{2}$ isochore does not cross the melt-crystal coexistence region on cooling. In contrast, a typical $P=P_{0}$ isobar does enter the melt-crystal coexistence region upon cooling from the melt, indicating the feasibility of crystallization at constant $P$. Viewed from a different perspective, in the two-phase region the total volume $V=X_{\mathrm{X}} V_{\mathrm{X}}{ }^{*}+X_{\mathrm{M}} V_{\mathrm{M}} *$ with $\delta X_{\mathrm{X}}=-\delta X_{\mathrm{M}}$, where $X_{\mathrm{M}}$ and $X_{\mathrm{X}}$ represent the number fraction of particles in the melt and crystal phase, respectively. Since $V_{\mathrm{X}} * \neq V_{\mathrm{M}}{ }^{*}$ in general, $\delta V \neq$ $0, v i z$. crystallization is not allowed at constant $V$. On the other hand, it is evident that $\delta V \neq 0$ can be accommodated under the condition of constant $P$. The conclusion that isobaric but not 
isochoric processes are allowed for ordering of hard spheres holds true for all hard particles, e.g. hard spherocylinders with a length-to-diamater ratio from 3 to $5{ }^{17}$ and hard oblates with a diameter-to-thickness ratio from 5 to $20^{27}$, which are capable of ordering transitions with similar equations of state and phase diagrams to those displayed in Figure 1 for hard spheres.

Furthermore, the previously reported calculations ${ }^{17,27}$ show that $\Delta V<0$ and hence $\Delta S<0$ under equilibrium ordering transitions to thermotroipc liquid crystals, consistent with our foregoing analysis of hard-sphere crystallization.
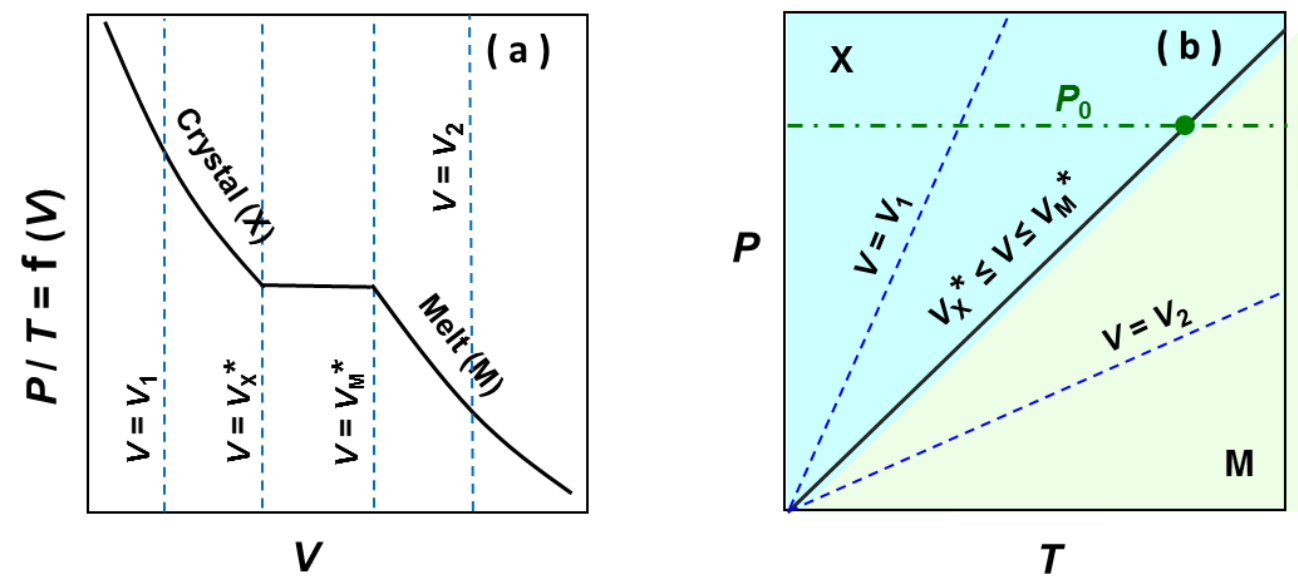

FIG. 1. Hard-sphere phase behavior: (a) The hard sphere equation of state plotted in $P / T$, adapted from Ref. ${ }^{23}$, as a function of $V$, in which $V_{1}, V_{2}, V_{\mathrm{X}} *$, and $V_{\mathrm{M}} *$ denote typical volumes of a closed system of fixed $N$ in the crystal and melt regions, and those on the verge of melting and crystallizing, respectively; and (b) The phase diagram derived from Part (a) with typical isochores as dashed lines in the crystal $\left(V_{1}\right)$ and melt $\left(V_{2}\right)$ region, the melt-crystal equilibrium represented by the solid line, and a typical isobar $\left(P_{0}\right)$ by the chain-dot line.

To drive a spontaneous process behind materials processing, the initial state must be out of equilibrium with the final state. On the Gibbs energy platform, $\Delta G<0$ follows from 
supercooling at constant $P$ or compression at constant $T$ from the melt-crystal equilibrium line into the crystal region as delineated in Figure $1 \mathrm{~b}$. The desired $\Delta G<0$ for process spontaneity requires that the reduction of the first term (i.e. enthalpy) outweigh the concomitant increase of the second term (i.e. due to entropy loss) in the expression, $\Delta G=P(\Delta V)-T(\Delta S)$. Therefore, spontaneous ordering transitions by supercooling at constant $P$ or compression at constant $T$ are indeed enthalpy-driven with both $\Delta H<0$ and $\Delta S<0$ derived from equilibrium consideration. Moreover, the same $\Delta H$ and $\Delta S$ values can be employed to determine the extent of perturbation in Gibbs energy, $\delta G=\Delta V \delta P-\Delta S \delta T$, from that on the coexistence line. Hence, the departure from equilibrium can be readily quantified in the Gibbs energy framework but not in the Helmholz energy framework.

Although several simulations at both constant $V^{28,29}$ and constant $P^{30-32}$ have shown spontaneous ordering upon perturbation from an equilibrium state, it is the nature of this perturbation - isobaric versus isochoric -- that ultimately determines whether a true equilibrium phase transition is at hand. That an equilibrium ordering transition is allowed at constant $P$ asserts that the Gibbs energy profile must possess two minima at the same level representing the initial and final states under equilibrium with each other. From the Helmholz energy perspective, there can be only one minimum state for a system held at constant $V$ because ordering transition between two states in equilibrium is forbidden. Unlike the initial less ordered phase, the final better ordered phase must incorporate all possible configurations with the same energy, each making an equal contribution, thereby maximizing $S$ to yield $\Delta S>0$. This is equivalent to the oft-quoted excluded-volume entropy effect, whereby an ordered structure improves excluded volume overlap to increase the number of ways of arranging hard particles. The inequality $\Delta A<0$ follows from the increase in combinatorial entropy associated with 
spontaneous ordering of hard particles at constant $V$. Glotzer et $a l .{ }^{6}$ did report a set of $\Delta S>0$ for the formation of liquid crystals and plastic crystals via self-assembly of hard particles, although the authors characterized their results as preliminary. To evaluate entropy-driven ordering at constant $V$, a remaining challenge is to definitively quantify the entropic gain originating in ordering from non-equilibrium initial states.

In summary, the driving forces behind hard-particle ordering transitions in the absence of solvent were evaluated using the Helmholz and Gibbs energy under constant $(V, T)$ and $(P, T)$, respectively. It is demonstrated that equilibrium ordering transitions are prohibited under the constraint of constant $V$. Existing computational and experimental results combine to rationalize equilibrium ordering transitions at constant $P$ accompanied by negative values of $\Delta V, \Delta H$ and $\Delta S$. It follows that spontaneous hard-particle ordering occurs when the Gibbs energy loss due to the pressure-volume effect outweighs the Gibbs energy gain due to a loss in entropy, namely, the process is enthalpy-driven. Whereas self-assembly of hard-body amphiphiles in complex fluids has been described theoretically as enthalpy-driven under constant $P,{ }^{33}$ recent experimental studies coupled with modeling have concluded that enthalpy competes with entropy behind depletion forces depending on the role that solvent plays and the departure from hard-core interaction ${ }^{34}$ In principle, ordering from initial states out of equilibrium with final states at constant $V$ appears feasible. While a volume reduction upon fast compression could plausibly induce ordering at constant $V$ through an entropy-driven process, processes at constant $P$ are far more common for which a classical thermodynamic framework can be readily established for driving process spontaneity. To formulate predictive materials processing strategies, further computations and experiments are warranted to elucidate particle ordering from initial, less ordered states progressing toward final, better ordered states at both constant $P$ and constant $V$. 
This demanding task requires quantifying the entropy of non-equilibrium initial states that falls under the purview of non-equilibrium statistical mechanics beyond the scope of this study.

\section{ACKNOWLEDGEMENTS}

The authors appreciate helpful discussions with their colleagues, Professors Andrew White, Matthew Z. Yates, and Eldred H. Chimowitz in the Chemical Engineering Department and Prof. Stephen Teitel in the Physics Department at the University of Rochester. Sandra Anthamatten's effort to improve readability of this manuscript is gratefully acknowledged. MA and SHC were supported in part by the Department of Energy Office of Inertial Confinement Fusion under Cooperative Agreement No. DE-FC52-08NA28302 with Laboratory for Laser Energetics at the University of Rochester. The support of DOE does not constitute an endorsement by DOE of the views expressed in this article.

\section{References}

1. Onsager, L. Ann Ny Acad Sci 1949, 51 (4), 627-659.

2. Wood, W. W.; Jacobson, J. D. J Chem Phys 1957, 27 (5), 1207-1208.

3. Alder, B. J.; Wainwright, T. E. J Chem Phys 1957, 27 (5), 1208-1209.

4. $\quad$ Palberg, T. J Phys-Condens Mat 2014, 26 (33), 333101.

5. Dijkstra, M. Adv Chem Phys 2015, 156, 35-71.

6. Damasceno, P. F.; Engel, M.; Glotzer, S. C. Science 2012, 337 (6093), 453-457.

7. Frenkel, D. Physica A 1999, 263 (1-4), 26-38.

8. Cheng, Z.; Chaikin, P. M.; Russel, W. B.; Meyer, W. V.; Zhu, J.; Rogers, R. B.; Ottewill, R. H. Mater Design 2001, 22 (7), 529-534.

9. $\quad$ Adams, M.; Dogic, Z.; Keller, S. L.; Fraden, S. Nature 1998, 393 (6683), 349-352. 
10. March, N. H.; Tosi, M. P., Introduction to liquid state physics. World Scientific Publishing: Singapore, 2002.

11. Mulero, A., Theory and simulation of hard-sphere fluids and related systems. Springer: Berlin, 2008; Vol. 753.

12. Lekkerkerker, H. N. W.; Tuinier, R., Colloids and the depletion interaction. In Lecture notes in physics, Springer Netherlands: 2011; Vol. 833.

13. Frenkel, D. Nat Mater 2015, 14 (1), 9-12.

14. Klotz, I. M.; Rosenberg, R. M., In Chemical thermodynamics: Basic concepts and methods, 7th ed.; Wiley: 2008.

15. Hoover, W. G.; Ree, F. H. J Chem Phys 1968, 49 (8), 3609-3617.

16. Vega, C.; Noya, E. G. J Chem Phys 2007, 127 (15).

17. McGrother, S. C.; Williamson, D. C.; Jackson, G. J Chem Phys 1996, 104 (17), 67556771.

18. Fischermeier, E.; Bartuschat, D.; Preclik, T.; Marechal, M.; Mecke, K. Comput Phys Commun 2014, 185 (12), 3156-3161.

19. Rutgers, M. A.; Dunsmuir, J. H.; Xue, J. Z.; Russel, W. B.; Chaikin, P. M. Phys Rev B 1996, 53 (9), 5043-5046.

20. Kamien, R. D.; Liu, A. J. Phys Rev Lett 2007, 99 (15).

21. Royall, C. P.; Poon, W. C. K.; Weeks, E. R. Soft Matter 2013, 9 (1), 17-27.

22. Lekkerkerker, H. N. W. T., R., Colloids and the depletion interaction. In Lecture notes in physics, Springer Netherlands: 2011; Vol. 833.

23. Alder, B. J.; Hoover, W. G.; Young, D. A. J Chem Phys 1968, 49 (8), 3688-3696.

24. Haynes, W. M., CRC handbook of chemistry and physics. 95th ed.; Boca Raton, Florida, 2014-15; p pp. 6-146 to 151.

25. Imhof, A.; Dhont, J. K. G. Phys Rev Lett 1995, 75 (8), 1662-1665.

26. Lago, S.; Cuetos, A.; Martinez-Haya, B.; Rull, L. F. J Mol Recognit 2004, 17 (5), 417425.

27. Wu, L.; Wensink, H. H.; Jackson, G.; Muller, E. A. Mol Phys 2012, 110 (11-12), 12691288.

28. van Anders, G.; Klotsa, D.; Ahmed, N. K.; Engel, M.; Glotzer, S. C. Proc Natl Acad Sci USA 2014, 111 (45), E4812-E4821. 
29. Schilling, T.; Dorosz, S.; Schope, H. J.; Opletal, G. J Phys-Condens Mat 2011, 23 (19), $1-5$.

30. Dogic, Z.; Frenkel, D.; Fraden, S. Phys Rev E 2000, 62 (3), 3925-3933.

31. Ni, R.; Gantapara, A. P.; de Graaf, J.; van Roij, R.; Dijkstra, M. Soft Matter 2012, 8 (34), 8826-8834.

32. Bolhuis, P.; Frenkel, D. J Chem Phys 1997, 106 (2), 666-687.

33. Henderson, J.R. Phys. Rev. E 1997, 55 (5), 5731-5742.

34. Sukenik, S.; Sapir, L.; Harries, D. Current Opinion in Colloid \& Interface Science 2013, 18, 495-501. 
TOC Graphic:

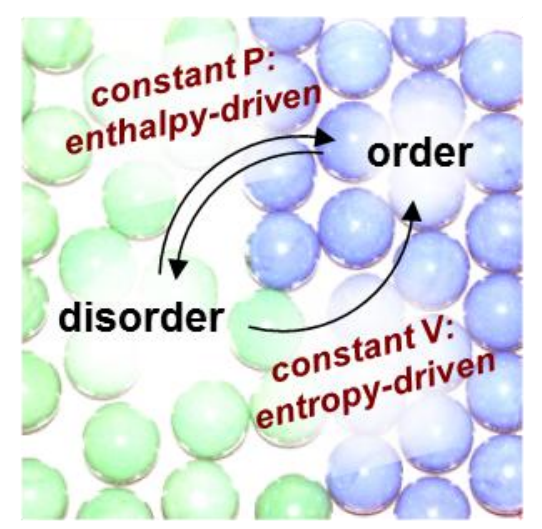

